\title{
Invariance entropy for outputs
}

\author{
Fritz Colonius · Christoph Kawan
}

\begin{abstract}
For continuous time control systems, this paper analyzes output invariance entropy as a measure for the information necessary to achieve invariance of compact subsets of the output space. For linear control systems with compact control range, relations to controllability and observability properties are studied. Furthermore, the notion of asymptotic output invariance entropy is introduced and characterized for these systems.
\end{abstract}

Keywords Nonlinear control $\cdot$ Invariance entropy · Outputs

Mathematics Subject Classification (2000) $\quad 94 \mathrm{~A} 17 \cdot 37 \mathrm{~B} 40 \cdot 93 \mathrm{C} 15$

\section{Introduction}

The motivation for this study comes from systems with restricted digital communication channels: if the controller cannot access the output of the system because of communication constraints, the information transferred to the controller becomes an issue. We will consider the problem to keep the output in a compact set $Q$. Then the exponential growth rate of the required number of control functions will give a lower bound on the required data rate. Since the number of controls for achieving this need not be finite, an appropriate mathematical formulation requires a slightly relaxed

Supported by DFG Grant Co 124/17-1 within DFG Priority Program 1305.

F. Colonius · C. Kawan $(\varangle)$

Institut für Mathematik, Universität Augsburg, 86135 Augsburg, Germany

e-mail: christoph.kawan@math.uni-augsburg.de

F. Colonius

e-mail: fritz.colonius@math.uni-augsburg.de 
notion, which allows for arbitrarily small deviations from the set $Q$. The motivation to consider open-loop controls in this context comes, in particular, from model predictive control (see, e.g., the collection [1] and [9], where optimal open-loop controls are computed and applied on short time intervals).

The problem to determine data rates needed in order to achieve desired control objectives has been around for quite a while. Early contributions are due to Wong and Brockett [17,18] and others. More recently, problems in networked control system have been treated, see, e.g., Gupta et al. [10], or Carli and Bullo [4] for quantized coordination algorithms for robots. In [15], Nair et al. introduced ideas from topological dynamics into this field. (See [11] for an authoritative presentation of the mathematical theory of dynamical systems.) They defined and studied topological feedback entropy for the problem of stabilizing a discrete-time system at an equilibrium.

Here, we follow our approach in [6] for invariance properties of subsets in the state space and generalize it to systems with outputs. We count how fast-for time $T$ tending to infinity - the number of open loop control functions grows which are needed in order to achieve desired properties of the system on $[0, T]$. For problems in the state space, a number of results for this notion can be proved; see, in addition to [6], also Kawan [12] where estimates for the invariance entropy are proven. The relations to topological feedback entropy and quantization are described in [6, Section 6]. The consequences for the required data rates (for problems in the state space) are also discussed in [5].

Our strategy here is to derive properties of the invariance entropy in the output space from properties of associated controlled invariant sets in the state space. Here, observability and also controllability properties will play a major role. In particular, for linear control systems the invariance entropy of subsets $Q$ of the state space with positive Lebesgue measure has been computed in [6] as the sum over all real parts of unstable eigenvalues. In the case with outputs, one will expect that unobservable modes have to be omitted, since they should not influence the invariance entropy for the output. Theorems 2 and 3 present sufficient conditions under which this conjecture holds true. In particular, Theorem 3 uses a special relation between the admissible initial values and the prescribed set in the output space. Furthermore, we introduce the notion of asymptotic output invariance entropy based on a definition which requires invariance only for time large enough. Here, for a set $K$ of initial values, we want to count the controls such that the corresponding outputs asymptotically approach a compact subset $Q$ in the output space (cf. Definition 6 and Theorem 5 for precise formulations). Then only the observable eigenvalues contribute to the asymptotic output invariance entropy, without the earlier restriction on the admissible sets of initial values.

We consider the present paper as a step towards a better understanding of the data rates needed to control interconnected systems which we hope to treat in the future. We also remark that in our opinion the machinery of topological entropy is a versatile tool, although at first sight it may appear rather heavy.

The contents of this paper are as follows: In Sect. 2, we collect some properties of observed linear control systems. Section 3 introduces invariance entropy for compact subsets of the output space of nonlinear systems and shows its invariance under topological conjugacies. In Sect. 4, the linear case is discussed in relation to observability and controllability properties. Here, it can be shown that only observable 
eigenvalues contribute to the output invariance entropy, provided that the admissible set of initial values is appropriately restricted. Finally, Sect. 5 presents results for asymptotic invariance entropy, again for linear control systems. Under an additional asymptotic controllability assumption to the unobservable subspace, it can be shown that the entropy is given by the sum of the real parts of the unstable controllable and observable eigenvalues.

Notation The closure and the interior of a set $A \subset \mathbb{R}^{d}$ are denoted by $\operatorname{cl} A$ and int $A$, respectively. The $\varepsilon$-neighborhood of $A$ is

$$
\mathbf{N}_{\varepsilon}(A):=\left\{x \in \mathbb{R}^{d}, \operatorname{dist}(x, A):=\inf _{a \in A}\|x-a\|<\varepsilon\right\} .
$$

\section{Preliminaries on linear control systems}

The purpose of this section is to recall some properties of linear control systems with output and to fix some notation.

We consider control systems in $\mathbb{R}^{d}$ of the form

$$
\dot{x}(t)=A x(t)+B u(t), y(t)=C x(t), \quad u \in \mathcal{U},
$$

with matrices $A \in \mathbb{R}^{d \times d}, B \in \mathbb{R}^{d \times m}$, and $C \in \mathbb{R}^{k \times d}$ and control range $U \subset \mathbb{R}^{m}$; the set $\mathcal{U}$ of admissible control functions is defined by

$$
\mathcal{U}=\left\{u \in L_{\infty}\left(\mathbb{R}, \mathbb{R}^{m}\right), u(t) \in U \text { for almost all } t \in \mathbb{R}\right\}
$$

The solution of the differential equation (1) with initial condition $x(0)=x_{0} \in \mathbb{R}^{d}$ and control $u \in \mathcal{U}$ is denoted by $\varphi\left(t, x_{0}, u\right)$. Here the relation $\varphi\left(t+t_{0}, x_{0}, u\right)=$ $\varphi\left(t, \varphi\left(t_{0}, x_{0}, u\right), u\left(t_{0}+\cdot\right)\right)$ holds. Using the variation-of-constants formula, the outputs are given by

$$
y(t)=C \varphi\left(t, x_{0}, u\right)=C e^{A t} x_{0}+\int_{0}^{t} C e^{A(t-s)} B u(s) \mathrm{d} s .
$$

The reachable sets from an initial point $x_{0} \in \mathbb{R}^{d}$ at time $t_{0}=0$ are

$$
\begin{aligned}
\mathcal{R}_{t}\left(x_{0}, U\right) & :=\left\{\varphi\left(t, x_{0}, u\right) \in \mathbb{R}^{d}, u \in \mathcal{U}\right\}, t \geq 0, \quad \text { and } \\
\mathcal{R}_{\leq T}\left(x_{0}, U\right) & :=\bigcup_{0 \leq t \leq T} \mathcal{R}_{t}\left(x_{0}, U\right) .
\end{aligned}
$$

The reachability subspace with unconstrained controls is

$$
\mathcal{R}:=\operatorname{Im}\left[B, A B, \ldots, A^{d-1} B\right]
$$


Using Cayley-Hamilton's Theorem, one sees that this subspace is the smallest $A$ invariant subspace containing $\operatorname{Im} B$ and that it coincides with the reachable set from the origin (at any time $t>0$ ) with unconstrained controls,

$$
\mathcal{R}=\left\{\varphi(t, 0, u) \in \mathbb{R}^{d}, u:[0, t] \rightarrow \mathbb{R}^{m} \text { continuous }\right\}
$$

We call the eigenvalues of $\left.A\right|_{\mathcal{R}}: \mathcal{R} \rightarrow \mathcal{R}$ the controllable eigenvalues.

The unobservable subspace $\mathcal{N}$ is

$$
\mathcal{N}=\bigcap_{i=0}^{d-1} \operatorname{ker} C A^{i}
$$

Again by Cayley-Hamilton's theorem, $\mathcal{N}$ is the largest $A$-invariant subspace contained in $\operatorname{ker} C$.

Consider the induced linear control system on $\mathbb{R}^{d} / \mathcal{N}$ (identified with $\mathbb{R}^{\bar{d}}$ ) given by $(\bar{A}, \bar{B}, \bar{C}) \in \mathbb{R}^{\bar{d} \times \bar{d}} \times \mathbb{R}^{\bar{d} \times m} \times \mathbb{R}^{k \times \bar{d}}$ and denote its trajectories by $\bar{\varphi}(t, \bar{x}, u)$. The natural projection $\mathbb{R}^{d} \rightarrow \mathbb{R}^{d} / \mathcal{N}=\mathbb{R}^{\bar{d}}$ is denoted by $\pi$. For an eigenvalue $\lambda \in \mathbb{C}$ of $A$ with real generalized eigenspace $E(\lambda)$, let $m_{\mathcal{N}}(\lambda)$ denote the dimension of $\pi(E(\lambda))$. We call the eigenvalues $\lambda$ of $\bar{A}$ the observable eigenvalues and $m_{\mathcal{N}}(\lambda)>0$ their observable multiplicities. System $(\bar{A}, \bar{B}, \bar{C})$ is observable, i.e., its unobservable subspace is trivial, and

$$
\operatorname{spec}(A)=\operatorname{spec}(\bar{A}) \cup \operatorname{spec}\left(\left.A\right|_{\mathcal{N}}\right)
$$

Since $\mathcal{R}$ is $A$-invariant, the subspace $\pi \mathcal{R} \subset \mathbb{R}^{d} / \mathcal{N}$ is $\bar{A}$-invariant and contains $\operatorname{Im} \bar{B}=$ $\pi \operatorname{Im} B$. It is the smallest subspace with these properties and hence it is the controllable subspace of $(\bar{A}, \bar{B}, \bar{C})$. The system obtained by restricting the observable system to its reachable subspace $\overline{\mathcal{R}}$ is controllable and observable. The eigenvalues of the corresponding map $\left.\bar{A}\right|_{\overline{\mathcal{R}}}$ are called the observable and controllable eigenvalues of $A$.

For observable $(A, C)$ the observability Gramians (see, e.g., [2], Corollary 3.2 and Corollary 3.8 in Chapter 3), defined by

$$
W\left(t_{0}, t_{1}\right):=\int_{t_{0}}^{t_{1}} e^{A^{*}\left(s-t_{0}\right)} C^{*} C e^{A\left(s-t_{0}\right)} \mathrm{d} s, \quad t_{1}>t_{0},
$$

are invertible. For the output $y(t)=C \varphi\left(t, x_{0}, u\right)$ and

$$
\hat{y}(t):=y(t)-\int_{0}^{t} C e^{A(t-s)} B u(s) \mathrm{d} s
$$


one has

$$
x_{0}=W(0, T)^{-1} \int_{0}^{T} e^{A^{*} s} C^{*} \hat{y}(s) \mathrm{d} s .
$$

This shows that the initial point is uniquely determined by the control function and the output function. In particular, for $u=0$ one has

$$
x_{0}=W(0, T)^{-1} \int_{0}^{T} e^{A^{*} s} C^{*} y(s) \mathrm{d} s .
$$

\section{Output invariance entropy}

In this section, we define output controlled invariant sets and a related notion of output invariance entropy. Then the behavior under conjugacies is characterized.

Consider a nonlinear control system with output

$$
\dot{x}=f(x, u(t)), y=g(x), \quad u \in \mathcal{U} \text {. }
$$

For simplicity, we assume that everything is defined in Euclidean spaces, i.e., $f$ : $\mathbb{R}^{d} \times \mathbb{R}^{m} \rightarrow \mathbb{R}^{d}$ and $g: \mathbb{R}^{d} \rightarrow \mathbb{R}^{k}$ are smooth (i.e., $C^{\infty}$ ), and

$$
\mathcal{U}=\left\{u \in L_{\infty}\left(\mathbb{R}, \mathbb{R}^{m}\right), \quad u(t) \in U \quad \text { for almost all } t \in \mathbb{R}\right\}
$$

with control range $U \subset \mathbb{R}^{m}$. The solution of the differential equation with initial condition $x(0)=x_{0} \in \mathbb{R}^{d}$ and control $u \in \mathcal{U}$ is denoted by $\varphi\left(t, x_{0}, u\right)$. We assume that unique global solutions exist.

Definition 1 A nonvoid subset $Q \subset \mathbb{R}^{k}$ is called output controlled invariant for system (3) if for all $y \in Q$ there are an initial state $x \in \mathbb{R}^{d}$ and a control $u \in \mathcal{U}$ with $g(x)=y$ and $g(\varphi(t, x, u)) \in Q$ for all $t \geq 0$. Then we denote

$$
P(Q):=\left\{x \in \mathbb{R}^{d}, \quad \text { there is } u \in \mathcal{U} \quad \text { with } g(\varphi(t, x, u)) \in Q \quad \text { for all } t \geq 0\right\} .
$$

Observe that $P(Q) \subset g^{-1}(Q)$ and that $Q$ is output controlled invariant iff $g(P(Q))=Q$. We want to describe how the number of (open-loop) control functions which are necessary to keep the system in $Q$ grows with time. This leads us to the following preliminary definition of an invariance entropy.

Definition 2 Let $Q \subset \mathbb{R}^{k}$ be an output controlled invariant set. For given $T>0$ we call a subset $\mathcal{S}^{*} \subset \mathcal{U}$ a $(T, Q)$-spanning set if for all $x \in P(Q)$ there is $u \in \mathcal{S}^{*}$ with

$$
g(\varphi(t, x, u)) \in Q \quad \text { for all } t \in[0, T] .
$$


By $r_{\text {inv }}^{*}(T, Q)$ we denote the minimal cardinality of a $(T, Q)$-spanning set. If no finite $(T, Q)$-spanning set exists, we set $r_{\text {inv }}^{*}(T, Q):=\infty$. The strict output invariance entropy $h_{\text {inv }}^{*}(Q)$ is defined by

$$
h_{\text {inv }}^{*}(Q):=\limsup _{T \rightarrow \infty} \frac{1}{T} \log r_{\text {inv }}^{*}(T, Q) .
$$

In general, we cannot guarantee that the strict output invariance entropy, or merely the numbers $r_{\text {inv }}^{*}(T, Q)$, are finite, see the following remark. Hence, we do not pursue this notion any further and instead discuss a relaxed notion.

Remark 1 As observed above, a set $\mathcal{S}^{*} \subset \mathcal{U}$ is $(T, Q)$-spanning iff for all $x$ in the subset $P(Q)$ of the state space there is $u \in \mathcal{S}^{*}$ with $\varphi(t, x, u) \in P(Q)$ for all $t \in[0, T]$. The dissertation [12] discusses, when for compact subsets of the state space such finite sets of control functions exist. Only in very special cases, e.g., for scalar systems, this property can be guaranteed.

Instead of discussing the preliminary notion specified above any further, we relax the condition on spanning sets of controls using the same device as in [6,12], now for the set $P(Q)$. Furthermore, a set $K$ of admissible initial states is specified.

Definition 3 Let $Q \subset \mathbb{R}^{k}$ be an output controlled invariant set and consider $K \subset$ $P(Q)$. For given $T, \varepsilon>0$ we call a subset $\mathcal{S} \subset \mathcal{U}$ a $(T, \varepsilon, K, Q)$-spanning set if for all $x \in K$ there is $u \in \mathcal{S}$ with

$$
\varphi(t, x, u) \in \mathbf{N}_{\varepsilon}(P(Q)) \quad \text { for all } t \in[0, T] .
$$

By $r_{\text {inv }}(T, \varepsilon, K, Q)$ we denote the minimal cardinality of a $(T, \varepsilon, K, Q)$-spanning set. If no finite $(T, \varepsilon, K, Q)$-spanning set exists, we set $r_{\text {inv }}(T, \varepsilon, K, Q):=\infty$.

In other words, we require for a $(T, \varepsilon, K, Q)$-spanning set $\mathcal{S}$ that for every initial value in $K$ there is a control in $\mathcal{S}$ such that up to time $T$ the trajectory remains in the $\varepsilon$-neighborhood of $P(Q)$. Recall that by controlled invariance $g(P(Q))=Q$. Now we consider what happens for $T \rightarrow \infty$ and $\varepsilon \rightarrow 0$ and define output invariance entropy.

Definition 4 Let $Q \subset \mathbb{R}^{k}$ be a compact output controlled invariant set and let $K \subset$ $P(Q)$. Then the output invariance entropy $h_{\mathrm{inv}}(K, Q)$ is defined by

$h_{\mathrm{inv}}(\varepsilon, K, Q):=\limsup _{T \rightarrow \infty} \frac{1}{T} \log r_{\mathrm{inv}}(T, \varepsilon, K, Q), h_{\mathrm{inv}}(K, Q):=\lim _{\varepsilon \searrow 0} h_{\mathrm{inv}}(\varepsilon, K, Q)$.

Note that $h_{\text {inv }}\left(\varepsilon_{1}, K, Q\right) \leq h_{\text {inv }}\left(\varepsilon_{2}, K, Q\right)$ for $\varepsilon_{2} \leq \varepsilon_{1}$. Hence, the limit for $\varepsilon \rightarrow 0$ exists (it may be infinite).

Remark 2 For systems with output $g=i d_{\mathbb{R}^{d}}$, the notions of output controlled invariance and $(T, \varepsilon, K, Q)$-spanning sets coincide with the corresponding notions in the state space introduced in [6]. We take this as a justification to use the same notation. Furthermore, if $K=P(Q)$, we omit the argument $K$ in the expressions introduced above.

Next we establish a number of consequences of the definitions. 
Proposition 1 Let $\mathcal{S} \subset \mathcal{U}$ be a $(T, \varepsilon, P(Q), Q)$-spanning set for a compact output controlled invariant set $Q \subset \mathbb{R}^{k}$. Then for every $y \in Q$ there is an initial state $x \in P(Q)$ with $g(x)=y$ and for all such $x$ there is a control $v \in \mathcal{S}$ with

$$
\operatorname{dist}(\varphi(t, x, v), P(Q))<\varepsilon \quad \text { for all } t \in[0, T]
$$

Proof By output controlled invariance, there is for $y \in Q$ a point $x \in P(Q)$ with $g(x)=y$. Then the assertion follows, since $\mathcal{S}$ is $(T, \varepsilon, P(Q), Q)$-spanning.

The next proposition specifies assumptions guaranteeing that the invariance entropy for outputs can be related to invariance entropy in the state space. Later, we will use Proposition 2 in order to compute the invariance entropy for linear control systems.

Proposition 2 Consider an output controlled invariant set $Q \subset \mathbb{R}^{k}$. Then $P(Q)$ is controlled invariant in the state space. If $Q$ is compact and $K$ is a compact subset of $P(Q)$, the output invariance entropy of $(K, Q)$ and the invariance entropy of $(K, P(Q))$ (for states) satisfy

$$
h_{\text {inv }}(K, P(Q))=h_{\text {inv }}(K, Q) \text {. }
$$

Suppose, additionally, that $g$ is uniformly continuous on a neighborhood of $P(Q)$. Then for every $\varepsilon>0$ there is $\delta>0$ such that every $(T, \delta, K, P(Q))$-spanning set $\mathcal{S}$ of controls has the following property: for every $x \in K$ there is $u \in \mathcal{S}$ with

$$
\operatorname{dist}(g(\varphi(t, x, u)), Q)<\varepsilon \text { for all } t \in[0, T]
$$

Proof Controlled invariance of $P(Q)$ follows, since for $x \in P(Q)$ there is $u \in \mathcal{U}$ with $g(\varphi(t, x, u)) \in Q$ for all $t \geq 0$. Hence, for all $T>0$ and $t \geq 0$ one has

$$
g(\varphi(t, \varphi(T, x, u), u(T+\cdot)))=g(\varphi(t+T, x, u)) \in Q
$$

which implies that $\varphi(T, x, u) \in P(Q)$ for all $T \geq 0$. The equality for the invariance entropies is immediate from the definitions. Finally, let $\varepsilon>0$. By uniform continuity of $g$ one finds $\delta>0$ such that in a neighborhood of $P(Q)$ the inequality $\left\|x_{1}-x_{2}\right\|<\delta$ implies $\left\|g\left(x_{1}\right)-g\left(x_{2}\right)\right\|<\varepsilon$. Thus, a $(T, \delta, K, P(Q))$-spanning set $\mathcal{S}$ of controls satisfies (4).

Invariance entropy is only interesting, if we can guarantee that for $T, \varepsilon>0$ there are finite $(T, \varepsilon)$-spanning sets. This holds under an additional assumption.

Lemma 1 Let $Q \subset \mathbb{R}^{k}$ be a compact set. Assume that for every bounded sequence $\left(x_{n}\right)$ in $\mathbb{R}^{d}$ and every sequence $\left(u_{n}\right)$ of controls in $\mathcal{U}$ there are $x_{0} \in \mathbb{R}^{d}$ and $u_{0} \in \mathcal{U}$ such that a subsequence of the trajectories $\varphi\left(t, x_{n}, u_{n}\right)$ converges uniformly on every compact interval $I \subset \mathbb{R}$ to $\varphi\left(t, x_{0}, u_{0}\right)$. 
(i) Suppose that for some $T>0$ the set

$P(Q, T):=\left\{x \in \mathbb{R}^{d}, \quad\right.$ there is $u \in \mathcal{U}$ with $g(\varphi(t, x, u)) \in Q$ for all $\left.t \in[0, T]\right\}$

is nonvoid and bounded. Then $P(Q, T)$ is compact.

(ii) Suppose, in addition to the assumption in (i), that $Q$ is output controlled invariant. Then $P(Q)$ is a nonvoid, compact, and controlled invariant subset of the state space and for all $\varepsilon>0$ there is a finite $(T, \varepsilon, P(Q), Q)$-spanning set.

Proof (i) By assumption the set $P(Q, T)$ is bounded. The set $P(Q, T)$ is closed, hence compact, by the compactness assumption for the trajectories.

(ii) Clearly, the set $P(Q)$ is nonvoid, compact, controlled invariant and contained in $P(Q, T)$. Let $\varepsilon>0$. By Proposition 1, for all $y \in Q$ there is an initial state $x \in P(Q)$ with $g(x)=y$ and for all such $x$ there is a control $u \in \mathcal{U}$ with $\varphi(t, x, u) \in P(Q)$ and $g(\varphi(t, x, u)) \in Q$ for all $t \geq 0$. By compactness of $P(Q)$ and continuous dependence on initial values it follows that there is a finite $(T, \varepsilon, P(Q), Q)$-spanning set $\mathcal{S} \subset \mathcal{U}$.

Remark 3 The compactness property in Lemma 1 is, in particular, satisfied for control-affine systems with compact and convex control range. This follows by a standard argument for existence of optimal controls (cf. [13, proof of Theorem 4 in Section 4.2] or [14, Section III.1]): The set of admissible control functions is weakly compact in $L_{2}\left([0, T], \mathbb{R}^{m}\right)$ and hence contains a weakly convergent subsequence. The corresponding trajectories converge uniformly on compact intervals.

Next we discuss the behavior of invariance entropy under semiconjugacy.

Theorem 1 Consider for $i=1,2$ two control systems of the form (3),

$$
\dot{x}_{i}=f_{i}\left(x_{i}, u_{i}(t)\right), y_{i}=g_{i}\left(x_{i}\right), \quad u_{i} \in \mathcal{U}_{i},
$$

in $\mathbb{R}^{d_{i}}$ with control ranges $U_{i} \subset \mathbb{R}^{m_{i}}$ and outputs $g_{i}: \mathbb{R}^{d_{i}} \rightarrow \mathbb{R}^{k_{i}}$. Let $\pi^{s}: \mathbb{R}^{d_{1}} \rightarrow \mathbb{R}^{d_{2}}$ and $\pi^{\text {out }}: \mathbb{R}^{k_{1}} \rightarrow \mathbb{R}^{k_{2}}$ be continuous maps and let $\pi^{\text {in }}: \mathcal{U}_{1} \rightarrow \mathcal{U}_{2}$ be any map. Denote the corresponding trajectories by $\varphi_{i}(t, x, u)$ and assume that the following semiconjugacy property holds for all $(t, x, u) \in[0, \infty) \times \mathbb{R}^{d_{1}} \times \mathcal{U}_{1}$ :

$$
\pi^{s}\left(\varphi_{1}(t, x, u)\right)=\varphi_{2}\left(t, \pi^{s}(x), \pi^{\text {in }}(u)\right), \pi^{\text {out }} \circ g_{1}=g_{2} \circ \pi^{s} .
$$

Let $Q \subset \mathbb{R}^{k_{1}}$ be a compact output controlled invariant set such that the restriction of $g_{1}$ to a neighborhood $\mathbf{N}_{\alpha}(P(Q)):=\left\{x \in \mathbb{R}^{d}\right.$, $\left.\operatorname{dist}(x, P(Q))<\alpha\right\}, \alpha>0$, is uniformly continuous. Then the following assertions hold:

(i) The set $\pi^{\text {out }}(Q) \subset \mathbb{R}^{k_{2}}$ is compact and output controlled invariant and for every compact subset $K \subset P(Q)$ the image $\pi^{s}(K)$ is compact and contained in $P\left(\pi^{\text {out }}(Q)\right)$ with

$$
h_{\text {inv }}(K, Q) \geq h_{\text {inv }}\left(\pi^{s}(K), \pi^{\text {out }}(Q)\right)
$$


(ii) Equality holds in (6) if, additionally, the map $\pi^{i n}: \mathcal{U}_{1} \rightarrow \mathcal{U}_{2}$ is surjective, and the maps $\pi^{s}$ and $\pi^{\text {out }}$ are homeomorphisms with $\left(\pi^{s}\right)^{-1}$ uniformly continuous on a neighborhood of $\pi^{s}(P(Q))$.

(iii) Semiconjugacy (5) holds, in particular, with $\pi^{i n}=$ id, if $\pi^{s}: \mathbb{R}^{d_{1}} \rightarrow \mathbb{R}^{d_{2}}$ is a continuously differentiable function with

$$
\begin{aligned}
D \pi^{s}(x) f_{1}(x, u) & =f_{2}\left(\pi^{s}(x), u\right) \quad \text { for all }(x, u) \in \mathbb{R}^{d_{1}} \times U_{1} \quad \text { and } \pi^{\text {out }} \circ \\
g_{1} & =g_{2} \circ \pi^{s} .
\end{aligned}
$$

Proof (i) Continuity of $\pi^{s}$ and $\pi^{\text {out }}$ imply that $\pi^{s}(K) \subset \mathbb{R}^{d_{2}}$ and $\pi^{\text {out }}(Q) \subset \mathbb{R}^{k_{2}}$ are compact sets. Semiconjugacy property (5) implies that $\pi^{\text {out }}(Q)$ is output controlled invariant. In fact: Let $y_{2} \in \pi^{\text {out }}(Q)$. Then there is $y_{1} \in Q$ with $\pi^{\text {out }}\left(y_{1}\right)=y_{2}$. Let $x_{1} \in \mathbb{R}^{d_{1}}$ and $u_{1} \in \mathcal{U}_{1}$ be such that $g_{1}\left(x_{1}\right)=y_{1}$ and $g_{1}\left(\varphi_{1}\left(t, x_{1}, u_{1}\right)\right) \in Q$ for all $t \geq 0$. Define $x_{2}:=\pi^{s}\left(x_{1}\right)$ and $u_{2}:=\pi^{i n}\left(u_{1}\right) \in \mathcal{U}_{2}$. Then the semiconjugacy condition implies for all $t \geq 0$

$$
\begin{aligned}
g_{2}\left(\varphi_{2}\left(t, x_{2}, u_{2}\right)\right) & =g_{2}\left(\varphi_{2}\left(t, \pi^{s}\left(x_{1}\right), \pi^{i n}\left(u_{1}\right)\right)\right) \\
& =g_{2}\left(\pi^{s}\left(\varphi_{1}\left(t, x_{1}, u_{1}\right)\right)\right) \\
& =\pi^{\text {out }}\left(g_{1}\left(\varphi_{1}\left(t, x_{1}, u_{1}\right)\right)\right) \in \pi^{\text {out }}(Q) .
\end{aligned}
$$

In particular, for $t=0$ one obtains $g_{2}\left(x_{2}\right)=\pi^{\text {out }}\left(g_{1}\left(x_{1}\right)\right)=\pi^{\text {out }}\left(y_{1}\right)=y_{2}$. The semiconjugacy condition also implies that

$$
\pi^{s}(K) \subset \pi^{s}(P(Q)) \subset P\left(\pi^{\text {out }}(Q)\right)
$$

In fact, the first inclusion is trivial. For the second inclusion, consider $x_{1} \in P(Q)$ and $u_{1} \in \mathcal{U}_{1}$ with $g_{1}\left(\varphi_{1}\left(t, x_{1}, u_{1}\right)\right) \in Q$ for all $t \geq 0$. Then

$g_{2}\left(\varphi_{2}\left(t, \pi^{s}\left(x_{1}\right), \pi^{\text {in }}\left(u_{1}\right)\right)\right)=\pi^{\text {out }}\left(g_{1}\left(\varphi_{1}\left(t, x_{1}, u_{1}\right)\right)\right) \in \pi^{\text {out }}(Q)$ for all $t \geq 0$,

and $\pi^{s}\left(x_{1}\right) \in P\left(\pi^{\text {out }}(Q)\right)$ follows. Now let $T, \varepsilon>0$. Since $\pi^{s}$ is uniformly continuous on a neighborhood $\mathbf{N}_{\alpha}(P(Q)), \alpha>0$, of the set $P(Q)$, there exists $\delta=\delta(\varepsilon)>0$ with

$$
\pi^{s}\left(\mathbf{N}_{\delta}(P(Q))\right) \subset \mathbf{N}_{\varepsilon}\left(\pi^{s}(P(Q))\right) \subset \mathbf{N}_{\varepsilon}\left(P\left(\pi^{\text {out }}(Q)\right)\right) .
$$

Let $\mathcal{S}_{1} \subset \mathcal{U}_{1}$ be a minimal $(T, \delta, K, Q)$-spanning set and define $\mathcal{S}_{2}:=\pi^{i n}\left(\mathcal{S}_{1}\right)$. Let $x_{2}=\pi^{s}\left(x_{1}\right) \in \pi^{s}(K)$ with $x_{1} \in K$. Then there is $u_{1} \in \mathcal{S}_{1}$ with $\varphi_{1}\left(t, x_{1}, u_{1}\right) \in$ $\mathbf{N}_{\delta}(P(Q))$ for all $t \in[0, T]$. Define $u_{2}:=\pi^{i n}\left(u_{1}\right)$. Then one finds for all $t \in[0, T]$

$$
\begin{aligned}
\varphi_{2}\left(t, x_{2}, u_{2}\right) & =\varphi_{2}\left(t, \pi^{s}\left(x_{1}\right), \pi^{i n}\left(u_{1}\right)\right) \\
& =\pi^{s}\left(\varphi_{1}\left(t, x_{1}, u_{1}\right)\right) \in \pi^{s}\left(\mathbf{N}_{\delta}(P(Q))\right) \subset \mathbf{N}_{\varepsilon}\left(P\left(\pi^{\text {out }}(Q)\right)\right) .
\end{aligned}
$$


This shows that $\mathcal{S}_{2}$ is a $\left(T, \varepsilon, \pi^{s}(K), \pi^{\text {out }}(Q)\right)$-spanning set. Hence, for every $\delta<\delta(\varepsilon)$ one has $r_{\text {inv }}(T, \delta, K, Q) \geq r_{\text {inv }}\left(T, \varepsilon, \pi^{s}(K), \pi^{\text {out }}(Q)\right)$ and inequality (6) follows.

(ii) First we prove that

$$
\pi^{s}(P(Q))=P\left(\pi^{\text {out }}(Q)\right)
$$

By (8), we only have to show that for $x_{2} \in P\left(\pi^{\text {out }}(Q)\right)$ there is $x_{1} \in P(Q)$ with $\pi^{s}\left(x_{1}\right)=x_{2}$. There is $u_{2} \in \mathcal{U}_{2}$ with $g_{2}\left(\varphi_{2}\left(t, x_{2}, u_{2}\right)\right) \in \pi^{\text {out }}(Q)$ for all $t \geq 0$. Then there are $x_{1} \in \mathbb{R}^{d_{1}}$ and $u_{1} \in \mathcal{U}_{1}$ with $\pi^{s}\left(x_{1}\right)=x_{2}$ and $\pi^{i n}\left(u_{1}\right)=u_{2}$. Hence, for all $t \geq 0$

$$
\begin{aligned}
\pi^{\text {out }}\left(g_{1}\left(\varphi_{1}\left(t, x_{1}, u_{1}\right)\right)\right) & =g_{2}\left(\pi^{s}\left(\varphi_{1}\left(t, x_{1}, u_{1}\right)\right)\right) \\
& =g_{2}\left(\varphi_{2}\left(t, \pi^{s}\left(x_{1}\right), \pi^{\text {in }}\left(u_{1}\right)\right)\right) \\
& =g_{2}\left(\varphi_{2}\left(t, x_{2}, u_{2}\right)\right) \in \pi^{\text {out }}(Q) .
\end{aligned}
$$

Since $\pi^{\text {out }}$ is a homeomorphism, it follows that $g_{1}\left(\varphi_{1}\left(t, x_{1}, u_{1}\right)\right) \in Q$ for all $t \geq 0$, and hence $x_{1} \in P(Q)$ proving (9). Now fix $\varepsilon, T>0$. Since $\left(\pi^{s}\right)^{-1}$ is uniformly continuous on a neighborhood $\mathbf{N}_{\alpha}\left(\pi^{s}(P(Q))\right), \alpha>0$, and equality (9) holds, there is $\delta>0$ with

$$
\left(\pi^{s}\right)^{-1}\left(\mathbf{N}_{\delta}\left(P\left(\pi^{\text {out }}(Q)\right)\right)\right)=\left(\pi^{s}\right)^{-1}\left(\mathbf{N}_{\delta}\left(\pi^{s}(P(Q))\right)\right) \subset \mathbf{N}_{\varepsilon}(P(Q)) .
$$

Let $\mathcal{S}_{2} \subset \mathcal{U}_{2}$ be a minimal $\left(T, \delta, \pi^{s}(K), \pi^{\text {out }}(Q)\right)$-spanning set and fix $x_{1} \in K$. Then

$$
x_{2}:=\pi^{s}\left(x_{1}\right) \in \pi^{s}(K) \subset \pi^{s}(P(Q))=P\left(\pi^{\text {out }}(Q)\right)
$$

and there is $u_{2} \in \mathcal{S}_{2}$ with

$$
\varphi_{2}\left(t, x_{2}, u_{2}\right) \in \mathbf{N}_{\delta}\left(P\left(\pi^{\text {out }}(Q)\right)\right) \quad \text { for all } t \in[0, T]
$$

Since $\pi^{i n}$ is surjective, we can pick $u_{1} \in \mathcal{U}_{1}$ with $\pi^{i n}\left(u_{1}\right)=u_{2}$. Define $\mathcal{S}_{1} \subset \mathcal{U}_{1}$ as the set of these controls $u_{1}$ and note that the number of elements in $\mathcal{S}_{1}$ coincides with the number of elements in $\mathcal{S}_{2}$. Then the semiconjugacy property implies for all $t \in[0, T]$

$$
\varphi_{1}\left(t, x_{1}, u_{1}\right)=\left(\pi^{s}\right)^{-1}\left(\varphi_{2}\left(t, \pi^{s}\left(x_{1}\right), \pi^{i n}\left(u_{1}\right)\right)\right) \in \mathbf{N}_{\varepsilon}(P(Q)) .
$$

Thus, $\mathcal{S}_{1}$ is $(T, \varepsilon, K, Q)$-spanning. This shows equality in (6).

(iii) Finally, suppose that $D \pi^{s}(x) f_{1}(x, u)=f_{2}\left(\pi^{s}(x), u\right)$ for all $(x, u) \in \mathbb{R}^{d_{1}} \times$ $U_{1}$. Then

$$
\pi^{s}\left(\varphi_{1}(0, x, u)\right)=\pi^{s}(x)
$$


and for almost all $t \geq 0$

$$
\begin{aligned}
\frac{\mathrm{d}}{\mathrm{d} t} \pi^{s}\left(\varphi_{1}(t, x, u)\right) & =D \pi^{s}\left(\varphi_{1}(t, x, u)\right) f_{1}\left(\varphi_{1}(t, x, u), u(t)\right) \\
& =f_{2}\left(\pi^{s}\left(\varphi_{1}(t, x, u)\right), u(t)\right)
\end{aligned}
$$

Hence, $\pi^{s}\left(\varphi_{1}(t, x, u)\right)$ coincides with $\varphi_{2}\left(t, \pi^{s}(x), u\right)$ for all $t \geq 0$. Together with $\pi^{\text {out }} \circ g_{1}=g_{2} \circ \pi^{s}$, this shows semiconjugacy (5).

\section{Output invariance entropy for linear systems}

In this section, we discuss the output invariance entropy of compact subsets in the output space for linear control system (1). Here controllability and observability properties play an essential role. The simple example (Example 1) of a two-dimensional system with different output maps illustrates the different cases that may occur here.

Throughout this section, we assume that the control range $U$ is compact and convex. We note the following consequence of Theorem 1 relating the entropy for (1) to the entropy for the induced observable system.

Lemma 2 Consider linear control system (1) and let $Q \subset \mathbb{R}^{k}$ be compact.

(i) If the set $Q$ is output controlled invariant for (1), then it is output controlled invariant for the induced observable system with state space $\mathbb{R}^{d} / \mathcal{N}$,

$$
\dot{z}(t)=\bar{A} z(t)+\bar{B} u(t), u \in \mathcal{U}, \bar{y}(t)=\bar{C} z(t) .
$$

(ii) Systems (1) and (10) are semiconjugate with the projection $\pi^{s}: \mathbb{R}^{d} \rightarrow \mathbb{R}^{d} / \mathcal{N}$ and identity maps $\pi^{\text {in }}$ on $\mathcal{U}$ and $\pi^{\text {out }}$ on $\mathbb{R}^{k}$. In particular, if the set $P(Q)$ for system (1) is compact, the output invariance entropy of $Q$ for system (1) is greater than or equal to the output invariance entropy of $Q$ for system (10).

Proof (i) Let $u \in \mathcal{U}$. Then for all $x \in \mathbb{R}^{d}$ one has

$$
C \varphi(t, x, u)=\bar{C} \bar{\varphi}\left(t, \pi^{s} x, u\right) .
$$

Hence, output controlled invariance of $Q$ for the induced observable system follows.

(ii) Here the assumptions of Theorem 1(i) are satisfied with $\pi^{\text {out }}$ and $\pi^{\text {in }}$ the identity maps and the surjective projection $\pi^{s}$. Thus, the inequality for the invariance entropies follows.

The following lemma shows that for a compact set $Q$ in the output space of an observable system, the set of initial values $x_{0} \in \mathbb{R}^{d}$ which lead to outputs in $Q$ on a finite interval is compact. Furthermore, we can always find finite spanning sets.

Lemma 3 Suppose that $(A, C)$ is observable and let $Q \subset \mathbb{R}^{k}$ be compact and output controlled invariant. 
(i) Then for every $T>0$ the set

$$
P(Q, T):=\left\{x \in \mathbb{R}^{d}, \text { there is } u \in \mathcal{U} \text { with } C \varphi(t, x, u) \in Q \text { for all } t \in[0, T]\right\}
$$

is compact.

(ii) The set $P(Q) \subset \mathbb{R}^{d}$ is compact and for all $T, \varepsilon>0$ there are finite $(T, \varepsilon, Q)$ spanning sets.

Proof (i) Let $T>0$ and pick $x \in \mathbb{R}^{d}$ and a control $u \in \mathcal{U}$. Then, by equation (2),

$$
\begin{aligned}
x & =W(0, T)^{-1} \int_{0}^{T} e^{A^{*} t} C^{*}\left[C \varphi(t, x, u)-\int_{0}^{t} C e^{A(t-s)} B u(s) \mathrm{d} s\right] \mathrm{d} t \\
& =W(0, T)^{-1}\left[\int_{0}^{T} e^{A^{*} t} C^{*} C \varphi(t, x, u) d t-\int_{0}^{T} \int_{0}^{t} C e^{A(t-s)} B u(s) \mathrm{d} s \mathrm{~d} t\right] .
\end{aligned}
$$

This shows that the set $P(Q, T)$ is bounded, since here $C \varphi(t, x, u) \in Q$ for all $t \in[0, T]$ and $Q$ and $U$ are bounded. Furthermore, since $U$ is compact and convex, system (1) satisfies the compactness assumption for the trajectories imposed in Lemma 1, see Remark 3.

(ii) By assertion (i), the set $P(Q, T)$ is compact. Thus, the assertion follows by Lemma 1(ii).

From [6, Theorem 4.1] we obtain the following characterization of the invariance entropy in the state space.

Proposition 3 Consider a linear control system with compact control range $U$ (without output, i.e., $C=I$ ) in $\mathbb{R}^{d}$,

$$
\dot{x}(t)=A x(t)+B u(t), \quad u \in \mathcal{U}
$$

Then for a compact controlled invariant subset $Q$ of the state space $\mathbb{R}^{d}$

$$
h_{\text {inv }}(Q) \leq \sum_{\lambda} \operatorname{Re} \lambda
$$

where the sum is taken over all positive real parts of the eigenvalues $\lambda$ of A counted with their multiplicities. If $Q$ has positive Lebesgue measure, then equality holds in (11).

Proof By Remark 2, the invariance entropy with $C=I_{d}$ defined above coincides with the state space entropy from [6]. Hence, the assertions follow from [6, Theorem 5.1].

In the next lemma we impose additional conditions ensuring a property in the reachable subspace. Note that one can restrict the state space of a control system (1) to its 
reachable subspace $\mathcal{R}$ and obtains

$$
\dot{x}=\left.A\right|_{\mathcal{R}} x+B u, y=\left.C\right|_{\mathcal{R}} x, \quad u \in \mathcal{U} .
$$

Lemma 4 Suppose that $\left.A\right|_{\mathcal{R}}$ is hyperbolic (i.e., there are no controllable eigenvalues on the imaginary axis), and assume that $0 \in \operatorname{int} U$ and $0 \in \operatorname{int} Q$. Then the set

$$
P_{\mathcal{R}}(Q):=\{x \in \mathcal{R}, \quad \text { there is a control } u \in \mathcal{U} \text { with } C \varphi(t, x, u) \in Q \text { for all } t \geq 0\}
$$

has nonvoid interior in the reachable subspace $\mathcal{R}$.

Proof For $\rho>0$, small enough, the control ranges

$$
U^{\rho}:=\left\{u \in \mathbb{R}^{m},\|u\| \leq \rho\right\}
$$

are contained in $U$. Denote the corresponding sets of admissible control functions by $\mathcal{U}^{\rho}$. Recall that a control set is a maximal set of approximate controllability: For the system with control range $U^{\rho}$, a set $D^{\rho}$ with nonvoid interior in the state space is a control set, if $D^{\rho} \subset \operatorname{cl} \bigcup_{t>0}\left[\mathcal{R}_{t}\left(x_{0}, U^{\varrho}\right)\right]$ for all $x_{0} \in D^{\rho}$ and $D^{\rho}$ is maximal with this property (see Example 1 for an illustration). Colonius and Spadini [8, Theorem 4.1] shows that for system (12) there exists a unique control set $D^{\rho}$ in $\mathcal{R} \subset \mathbb{R}^{d}$ and $0 \in \operatorname{int} D^{\rho}$, where the interior is taken with respect to $\mathcal{R}$. Furthermore, for $\rho \rightarrow 0$, the diameter of $\operatorname{cl} D^{\rho}$ shrinks to zero. The initial point $x_{0}=0$ is in $P_{\mathcal{R}}(Q)$, since $C \varphi(t, 0,0)=0 \in \operatorname{int} Q$ for all $t \geq 0$. Since $U^{\rho}$ is bounded, there is $T>0$, such that $C \varphi(t, 0, u) \in Q$ for all $t \in[0, T]$ and all $u \in \mathcal{U}^{\rho}$. We may take $T>0$ small enough such that

$$
\mathcal{R}_{\leq T}\left(0, U^{\rho}\right):=\left\{\varphi(t, 0, u), 0 \leq t \leq T \text { and } u \in \mathcal{U}^{\rho}\right\} \subset \operatorname{int} D^{\rho} \subset \mathcal{R}
$$

In int $D^{\rho}$ exact reachability holds (cf. [8, Lemma 2.1] or Colonius and Kliemann [7, Lemma 3.2.13]). Hence, every point in $\mathcal{R}_{\leq T}\left(0, U^{\rho}\right)$ can be steered back to the origin, naturally without leaving $D^{\rho}$. Since $0=C 0 \in \operatorname{int} Q$ and the map $C$ is continuous, one may take $\rho>0$ small enough, such that all points on such a trajectory are mapped by $C$ into $Q$. Extending the controls periodically to $[0, \infty)$ one sees that $\mathcal{R}_{\leq T}\left(0, U^{\rho}\right) \subset P_{\mathcal{R}}(Q)$. The small time reachable set $\mathcal{R}_{\leq T}\left(0, U^{\rho}\right)$ has nonvoid interior in the subspace $\mathcal{R}$, hence $P_{\mathcal{R}}(Q)$ has nonvoid interior in $\mathcal{R}$, as claimed.

For the following result recall that the induced observable system associated with (1) is given by the matrices $(\bar{A}, \bar{B}, \bar{C})$ and controls in $\mathcal{U}$.

Theorem 2 Consider system (1) where the control range $U$ is compact and convex with $0 \in \operatorname{int} U$ and let $Q \subset \mathbb{R}^{k}$ be a compact output controlled invariant set with $0 \in \operatorname{int} Q$.

(i) Suppose that $(A, C)$ is observable. Then the set

$$
P(Q)=\left\{x \in \mathbb{R}^{d}, \quad \text { there is } u \in \mathcal{U} \text { with } C \varphi(t, x, u) \in Q \text { for all } t \geq 0\right\}
$$


is compact and the output invariance entropy of $Q$ satisfies the inequality

$$
h_{\text {inv }}(Q) \leq \sum_{\lambda} \operatorname{Re} \lambda,
$$

where the sum on the right-hand side is taken over all eigenvalues $\lambda$ of $A$ with positive real parts.

(ii) Suppose that $(A, B)$ is controllable, that the matrix $A$ is hyperbolic, and that the set $P(Q)$ is compact. Then

$$
h_{\text {inv }}(Q) \geq \sum_{\lambda} \operatorname{Re} \lambda,
$$

where the sum on the right-hand side is taken over all eigenvalues $\lambda$ of $A$ with positive real parts.

(iii) If $(A, C)$ is observable and $(A, B)$ is controllable with a hyperbolic matrix $A$, then equality holds in (14).

Proof (i) By observability, Lemma 3(ii) implies that the set $P(Q)$ in (13) is compact. Furthermore, this set is controlled invariant in the state space. Proposition 3 shows that the invariance entropy in the state space satisfies

$$
h_{\text {inv }}(P(Q)) \leq \sum_{\lambda} \operatorname{Re} \lambda,
$$

where summation is over the eigenvalues $\lambda$ of $A$ with positive real parts. Finally, the equality

$$
h_{\text {inv }}(P(Q))=h_{\text {inv }}(Q),
$$

follows from Proposition 2.

(ii) By Lemma 2, the invariance entropy $h_{\text {inv }}(Q)$ of $Q$ for system (1) is greater than or equal to the invariance entropy of $Q$ for the induced observable system. It is easily seen that this system is also reachable. Hence, we may assume without loss of generality that $(A, C)$ is observable and $(A, B)$ is reachable. By Lemma 4 and reachability, the set $P(Q)$ has nonvoid interior. Thus, Proposition 3 shows that equality holds in (15).

(iii) This is immediate from assertions (i) and (ii) noting that observability implies compactness of $P(Q)$.

The following simple example illustrates some of the results above.

Example 1 Consider the two-dimensional system $(d=2, m=k=1)$

$$
\left(\begin{array}{l}
\dot{x}_{1} \\
\dot{x}_{2}
\end{array}\right)=\left(\begin{array}{rr}
1 & 0 \\
0 & -1
\end{array}\right)\left(\begin{array}{l}
x_{1} \\
x_{2}
\end{array}\right)+\left(\begin{array}{l}
1 \\
1
\end{array}\right) u(t),
$$


and controls satisfying $u(t) \in U=[-1,1]$. The solutions are

$$
\left(\begin{array}{l}
x_{1}(t) \\
x_{2}(t)
\end{array}\right)=\left(\begin{array}{c}
e^{t} x_{1}^{0} \\
e^{-t} x_{2}^{0}
\end{array}\right)+\int_{0}^{t} u(s)\left(\begin{array}{c}
e^{t-s} \\
e^{s-t}
\end{array}\right) \mathrm{d} s .
$$

Inspection of the phase portraits for $u \equiv 1$ and $u \equiv-1$ shows that there is a unique control set $D$ with nonvoid interior,

$$
D=(-1,1) \times[-1,1]
$$

and $h_{\mathrm{inv}}(\mathrm{cl} D)=1$. The system is reachable, since the matrix

$$
(B, A B)=\left(\begin{array}{rr}
1 & 1 \\
1 & -1
\end{array}\right)
$$

has rank $d=2$. Next we discuss three different output maps.

(i) The system with output matrix $C=(1,1)$, i.e.,

$$
y=(1,1)\left(\begin{array}{l}
x_{1} \\
x_{2}
\end{array}\right)=x_{1}+x_{2},
$$

is observable, since the matrix

$$
\left(\begin{array}{c}
C \\
C A
\end{array}\right)=\left(\begin{array}{rr}
1 & 1 \\
1 & -1
\end{array}\right)
$$

has rank $d=2$. Clearly, $Q:=C(\operatorname{cl} D)=\left\{x_{1}+x_{2}, x_{1}, x_{2} \in[-1,1]\right\}=[-2,2]$ and $\left\{x \in \mathbb{R}^{2}, C x \in Q\right\}=\left\{\left(x_{1}, x_{2}\right),\left|x_{1}+x_{2}\right| \leq 2\right\}$ is unbounded, since ker $C$ is nontrivial. By Theorem 2, it follows that

$$
h_{\text {inv }}([-2,2])=h_{\text {inv }}([-1,1] \times[-1,1])=1 .
$$

By observability, the set $P(Q)$ is bounded. This follows from Lemma 3, since already the set $P(Q, T)$ of points $x$ with $C \varphi(t, x, u) \in Q$ on any interval $[0, T]$ is bounded. Observe that for $x_{0} \in \operatorname{ker} C$, the trajectory $\varphi\left(t, x_{0}, 0\right), t>0$, immediately leaves ker $C$.

(ii) The system with output matrix $C=(0,1)$, i.e.,

$$
y=(0,1)\left(\begin{array}{l}
x_{1} \\
x_{2}
\end{array}\right)=x_{2},
$$

is not observable. Here for $Q:=C(\operatorname{cl} D)=[-1,1]$ one obtains $\left\{x \in \mathbb{R}^{2}, C x \in\right.$ $Q\}=\mathbb{R} \times[-1,1]$, and

$$
P(Q)=[-1,1] \times[-1,1]
$$

is compact. 
(iii) Similarly, the system with output matrix $C=(1,0)$, i.e.,

$$
y=(1,0)\left(\begin{array}{l}
x_{1} \\
x_{2}
\end{array}\right)=x_{1},
$$

is not observable. Again for $Q=[-1,1]$, one obtains $\left\{x \in \mathbb{R}^{2}, C x \in Q\right\}=$ $[-1,1] \times \mathbb{R}$, and the set $P(Q)$ is unbounded and given by

$$
P(Q)=[-1,1] \times \mathbb{R} .
$$

Observe that this system has the observable eigenvalue $\lambda=1$ with positive real part.

Next we discuss invariance entropy for systems where the set $P(Q)$ need not be bounded. Example 1(iii) shows that for linear systems which are not observable, the set $P(Q)$ may be noncompact. In this situation, in order to obtain that only the observable eigenvalues contribute to the invariance entropy, we consider special (noncompact) sets $K \subset P(Q)$ of initial values. The following theorem estimates the entropy of nonobservable systems in such a case.

Theorem 3 Consider system (1), where the control range $U$ is compact and convex with $0 \in U$ and let $Q \subset \mathbb{R}^{k}$ be a compact output controlled invariant neighborhood of the origin. Let $Q_{0}$ and $U_{0}$ be compact neighborhoods of the origin in $\mathbb{R}^{k}$ and $\mathbb{R}^{m}$, respectively, with

$$
Q_{0}+Q_{0} \subset Q \text { and } U_{0}+U_{0} \subset U \text {. }
$$

Let $\mathcal{U}_{0}:=\left\{u_{0} \in \mathcal{U}, u_{0}(t) \in U_{0}\right.$ for all $\left.t \geq 0\right\}$ and define

$$
K:=\left\{x \in \mathbb{R}^{d}, \quad \text { there is } u_{0} \in \mathcal{U}_{0} \quad \text { with } C \varphi\left(t, x, u_{0}\right) \in Q_{0} \quad \text { for all } t \geq 0\right\}
$$

Then the output invariance entropy satisfies

$$
h_{\mathrm{inv}}(K, Q) \leq \sum_{\lambda} \operatorname{Re} \lambda
$$

where the sum on the right-hand side is taken over all observable eigenvalues $\lambda$ with positive real parts; i.e., the eigenvalues of $\bar{A}$ with positive real parts.

Proof Recall from Sect. 2 that $\mathcal{N}$ is the unobservable subspace. Consider the linear flow $\bar{\Phi}(t, x)=e^{\bar{A} t} x, \bar{\Phi}: \mathbb{R} \times \mathbb{R}^{d} / \mathcal{N} \rightarrow \mathbb{R}^{d} / \mathcal{N}$. With respect to any norm $\|\cdot\|$ on $\mathbb{R}^{d} / \mathcal{N}$, this flow satisfies the following uniform continuity condition: for all $t_{0}>0$, $t \in\left[0, t_{0}\right]$, and $x_{1}, x_{2} \in \mathbb{R}^{d} / \mathcal{N}$ one has

$$
\left\|e^{\bar{A} t} x_{1}-e^{\bar{A} t} x_{2}\right\|=\left\|e^{\bar{A} t}\left(x_{1}-x_{2}\right)\right\| \leq\left\|e^{\bar{A} t}\right\|\left\|x_{1}-x_{2}\right\| \leq\left(\max _{t \in\left[0, t_{0}\right]}\left\|e^{\bar{A} t}\right\|\right)\left\|x_{1}-x_{2}\right\|
$$


Hence (cf. [6, Lemma 2.1]), the topological entropy $h_{\text {top }}(\bar{\Phi})$ equals the topological entropy of the time-one-map $\bar{\Phi}_{1}(x)=e^{\bar{A}} x$. Recall from Bowen [3] (cf. also Katok and Hasselblatt [11] or Robinson [16]) that the topological entropy of a linear map $\Psi$ on $\mathbb{R}^{d}$ can be defined in the following way: For a compact set $K \subset \mathbb{R}^{d}$, numbers $n \in \mathbb{N}$ and $\varepsilon>0$ an $(n, \varepsilon, K, \Psi)$-spanning set is a subset $R \subset \mathbb{R}^{d}$ such that for all $x \in K$ there is $y \in R$ with $\left\|\Psi^{i}(x)-\Psi^{i}(y)\right\|<\varepsilon$ for all $i \in\{0,1, \ldots, n-1\}$. Similarly, a subset $S \subset K$ is called $(n, \varepsilon, K, \Psi)$-separated if for all $x, y \in S, x \neq y$, there is $i \in\{0,1, \ldots, n-1\}$ with $\left\|\Psi^{i}(x)-\Psi^{i}(y)\right\| \geq \varepsilon$. Denote the minimal cardinality of an $(n, \varepsilon, K, \Psi)$-spanning set by $r(n, \varepsilon, K, \Psi)$ and the maximal cardinality of an $(n, \varepsilon, K, \Psi)$-separated set by $s(n, \varepsilon, K, \Psi)$. Then

$h_{\text {top }}(K, \Psi):=\lim _{\varepsilon \rightarrow 0} \limsup _{n \rightarrow \infty} \frac{1}{n} \log r(n, \varepsilon, K, \Psi)=\lim _{\varepsilon \rightarrow 0} \limsup _{n \rightarrow \infty} \frac{1}{n} \log s(n, \varepsilon, K, \Psi)$,

and

$$
h_{\text {top }}(\Psi):=\sup _{K} h_{\text {top }}(K, \Psi)
$$

where the supremum is taken over all compact $K \subset \mathbb{R}^{d}$. By Bowen [3] the topological entropy of the linear map $\bar{\Phi}_{1}$ on $\mathbb{R}^{d} / \mathcal{N}$ is given by

$$
h_{\mathrm{top}}\left(\bar{\Phi}_{1}\right)=\sum_{i:\left|v_{i}\right|>1} \log \left|v_{i}\right|
$$

where $v_{1}, \ldots, v_{\bar{d}}$ are the eigenvalues of $e^{\bar{A}}$ (repeated according to their multiplicities.) Here $\mathbb{R}^{d} / \mathcal{N}$ is endowed with the metric

$$
\mathrm{d}(x+\mathcal{N}, y+\mathcal{N})=\inf _{z \in \mathcal{N}}\|x-y-z\|
$$

Since $\left|\nu_{i}\right|=\left|e^{\lambda_{i}}\right|=e^{\operatorname{Re} \lambda_{i}}$, where $\lambda_{1}, \ldots, \lambda_{\bar{d}}$ are the eigenvalues of $\bar{A}$, we obtain

$$
h_{\text {top }}(\bar{\Phi})=h_{\text {top }}\left(\bar{\Phi}_{1}\right)=\sum_{i:\left|e^{\lambda_{i}}\right|>1} \operatorname{Re} \lambda_{i}=\sum_{i: \operatorname{Re} \lambda_{i}>0} \operatorname{Re} \lambda_{i}
$$

Denote the natural projection of $\mathbb{R}^{d}$ to $\mathbb{R}^{d} / \mathcal{N}$ by $\pi$ and define $\bar{K}:=\pi(K)$. Since for all $t \geq 0, x \in \mathbb{R}^{d}$, and $u \in \mathcal{U}$ one has $C \varphi(t, x, u)=\bar{C} \bar{\varphi}(t, \pi x, u)$, it follows that

$$
\bar{K}=\left\{\pi x \in \mathbb{R}^{d} / \mathcal{N}, \text { there is } u_{0} \in \mathcal{U}_{0} \text { with } \bar{C} \bar{\varphi}\left(t, \pi x, u_{0}\right) \in Q_{0} \text { for all } t \geq 0\right\}
$$

By observability and Lemma 3 the set $\bar{K} \subset \mathbb{R}^{d} / \mathcal{N}$ is compact. We also observe that $\mathcal{N} \subset K$, since for $x \in \mathcal{N}$ one has $C \varphi(t, x, 0)=0 \in Q_{0}$ for all $t \geq 0$. For $x_{1}, x_{2} \in K$ there are controls $u_{1}, u_{2} \in \mathcal{U}_{0}$ such that for $t \geq 0$ 


$$
\begin{aligned}
C \varphi\left(t, x_{1}+x_{2}, u_{1}+u_{2}\right) & =C \varphi\left(t, x_{1}, u_{1}\right)+C \varphi\left(t, x_{2}, u_{2}\right) \in Q_{0}+Q_{0} \subset Q, \\
u_{1}(t)+u_{2}(t) & \in U_{0}+U_{0} \subset U, \text { hence } u_{1}+u_{2} \in \mathcal{U}
\end{aligned}
$$

This shows that

$$
K+K \subset P(Q)
$$

Now fix $T, \varepsilon>0$ and let $E \subset \bar{K}=\pi(K)$ be a maximal $(T, \varepsilon, \bar{K}, \bar{\Phi})$-separated set with respect to the flow $\bar{\Phi}$ on $\mathbb{R}^{d} / \mathcal{N}$, say $E=\left\{\pi x_{1}, \ldots, \pi x_{n}\right\}$ with $x_{1}, \ldots, x_{n} \in K$, and $n:=s(T, \varepsilon, \bar{K}, \bar{\Phi})$. Then $E$ is also $(T, \varepsilon, \bar{K}, \bar{\Phi})$-spanning for $\bar{\Phi}$, which means that for every $x \in K$ there is $x_{j}, j \in\{1, \ldots, n\}$, with

$$
\begin{aligned}
\max _{t \in[0, T]} \mathrm{d}\left(e^{\bar{A} t} \pi x, e^{\bar{A} t} \pi x_{j}\right) & =\max _{t \in[0, T]} \operatorname{dist}\left(e^{A t} x-e^{A t} x_{j}, \mathcal{N}\right) \\
& =\max _{t \in[0, T]} \inf _{z \in \mathcal{N}}\left\|e^{A t} x-e^{A t} x_{j}-z\right\|<\varepsilon
\end{aligned}
$$

The set $K$ is controlled invariant with respect to controls in $\mathcal{U}_{0}$. Hence, we can assign to each $x_{j} \in K, j=1, \ldots, n$, a control function $u_{j} \in \mathcal{U}_{0}$ such that $\varphi\left([0, \infty), x_{j}, u_{j}\right) \subset$ $K$. Let $\mathcal{S}:=\left\{u_{1}, \ldots, u_{n}\right\} \subset \mathcal{U}_{0}$. Using $\mathcal{N} \subset K$ and linearity, we obtain that for all $x \in K$ there is $j$ such that

$$
\begin{gathered}
\max _{t \in[0, T]} \operatorname{dist}\left(\varphi\left(t, x, u_{j}\right)-\varphi\left(t, x_{j}, u_{j}\right), K\right) \leq \max _{t \in[0, T]} \operatorname{dist}\left(\varphi\left(t, x, u_{j}\right)\right. \\
\left.-\varphi\left(t, x_{j}, u_{j}\right), \mathcal{N}\right)=\max _{t \in[0, T]} \operatorname{dist}\left(e^{A t} x-e^{A t} x_{j}, \mathcal{N}\right)<\varepsilon .
\end{gathered}
$$

Since $\varphi\left(t, x_{j}, u_{j}\right) \in K$ for all $t \in[0, T]$ and $K+K \subset P(Q)$, this implies that for all $x \in K$ there is $u_{j} \in \mathcal{S}$ such that

$$
\max _{t \in[0, T]} \operatorname{dist}\left(\varphi\left(t, x, u_{j}\right), P(Q)\right) \leq \max _{t \in[0, T]} \operatorname{dist}\left(\varphi\left(t, x, u_{j}\right), K+K\right)<\varepsilon
$$

This shows that $\mathcal{S}$ is $(T, \varepsilon, K, Q)$-spanning and hence

$$
r_{\text {inv }}(T, \varepsilon, K, Q) \leq s(T, \varepsilon, \pi(K), \bar{\Phi}) \quad \text { for all } T, \varepsilon>0 \text {, }
$$

and consequently

$$
h_{\text {inv }}(K, Q) \leq h_{\text {top }}(\pi(K), \bar{\Phi}) \leq h_{\text {top }}(\hat{\Phi})=\sum_{i: \operatorname{Re} \lambda_{i}>0} \operatorname{Re} \lambda_{i}
$$

This completes the proof of Theorem 3. 


\section{Asymptotic invariance entropy}

In the following, we propose a modified version of invariance entropy. We weaken the assumption that spanning sets of controls keep the system near the set $P(Q)$ for all times. Instead, we only require this for all times large enough. This may appear reasonable from a control-theoretic point of view and also, since entropy is an asymptotic property. This will allow us to deal with unbounded states leading to outputs in $Q$, without the additional assumptions imposed in Theorem 3 on the set of admissible initial values $K$. More precisely, we introduce the following notions.

Definition 5 Let $Q \subset \mathbb{R}^{k}$ be an output controlled invariant set of system (3). For a set $K \subset \mathbb{R}^{d}$ and $\varepsilon>0$ fix times $T>T_{0} \geq 0$. We call a subset $\mathcal{S} \subset \mathcal{U}$ a $\left(T, T_{0}, \varepsilon, K, Q\right)$ spanning set if for all $x \in K$ there is $u \in \mathcal{S}$ with

$$
\operatorname{dist}(\varphi(t, x, u), P(Q))<\varepsilon \quad \text { for all } t \in\left[T_{0}, T\right] .
$$

By $r_{\mathrm{as}}\left(T, T_{0}, \varepsilon, K, Q\right)$ we denote the minimal cardinality of a $\left(T, T_{0}, \varepsilon, K, Q\right)$ spanning set. If no finite $\left(T, T_{0}, \varepsilon, K, Q\right)$-spanning set exists, we set $r_{\mathrm{as}}\left(T, T_{0}\right.$, $\varepsilon, K, Q):=\infty$.

In other words: we require for a $\left(T, T_{0}, \varepsilon, K, Q\right)$-spanning set $\mathcal{S}$ that for every initial value in $K \subset \mathbb{R}^{d}$, there is a control in $\mathcal{S}$ such that for all times $t$ between $T_{0}$ and $T$ the trajectory remains in the $\varepsilon$-neighborhood of $P(Q)$. Now we consider what happens for $T \rightarrow \infty$, then $T_{0} \rightarrow \infty$, and, finally, $\varepsilon \rightarrow 0$ and obtain the following variant of invariance entropy.

Definition 6 Let $Q \subset \mathbb{R}^{k}$ be a compact output controlled invariant set and let $K \subset \mathbb{R}^{d}$. Then the asymptotic output invariance entropy $h_{\mathrm{as}}(K, Q)$ is defined by

$$
\begin{aligned}
h_{\mathrm{as}}(\varepsilon, K, Q) & :=\lim _{T_{0} \rightarrow \infty} \limsup _{T \rightarrow \infty} \frac{1}{T} \log r_{\mathrm{as}}\left(T, T_{0}, \varepsilon, K, Q\right), \\
h_{\mathrm{as}}(K, Q) & :=\lim _{\varepsilon \searrow 0} h_{\mathrm{as}}(\varepsilon, K, Q) .
\end{aligned}
$$

The expression $h_{\text {as }}(\varepsilon, K, Q)$ is well defined, since the limit for $T_{0} \rightarrow \infty$ exists: For $T_{0}>T_{1}$ every $\left(T, T_{1}, \varepsilon, K, Q\right)$-spanning set is also $\left(T, T_{0}, \varepsilon, K, Q\right)$-spanning, hence $r_{\text {as }}\left(T, T_{0}, \varepsilon, K, Q\right) \leq r_{\text {as }}\left(T, T_{1}, \varepsilon, K, Q\right)$ and, by monotonicity of the logarithm, the limit for $T_{0} \rightarrow \infty$ equals the infimum. It is also immediate from the definition that the asymptotic output invariance entropy is not greater than the output invariance entropy. Note that for systems without output, i.e., $g=\mathrm{id}$, and a controlled invariant set $Q \subset \mathbb{R}^{d}$, one has $P(Q)=Q$ and one obtains a notion of asymptotic invariance entropy in the state space. Finally, note that for $K_{1} \subset K_{2}$ one has $h_{\text {as }}\left(K_{1}, Q\right) \leq h_{\text {as }}\left(K_{2}, Q\right)$.

For linear control systems we obtain the following estimate from above, without observability assumption. Instead we require an asymptotic controllability condition to the unobservable subspace. 
Theorem 4 Consider system (1), where the control range $U$ is compact and convex with $0 \in U$, let $Q \subset \mathbb{R}^{k}$ be a compact output controlled invariant set with $0 \in Q$ and fix a compact subset $K \subset \mathbb{R}^{d}$. Assume that for all $\varepsilon>0$ there is a time $T_{0}(\varepsilon) \geq 0$ such that for all $x \in K$ there is a control $u \in \mathcal{U}$ with

$$
\operatorname{dist}(\varphi(t, x, u), \mathcal{N})<\varepsilon \text { for all } t \geq T_{0}(\varepsilon)
$$

Then the asymptotic output invariance entropy satisfies

$$
h_{\mathrm{as}}(K, Q) \leq \sum_{\lambda} \operatorname{Re} \lambda,
$$

where the sum on the right-hand side is taken over all observable eigenvalues $\lambda$ with positive real parts; i.e., the eigenvalues of $\bar{A}$ with positive real parts.

Proof The proof proceeds along the lines of Theorem 3. Now $K$ is a compact subset of $\mathbb{R}^{d}$ and for the induced flow $\bar{\Phi}$, the topological entropy of the compact set $\bar{K}=\pi(K) \subset \mathbb{R}^{d} / \mathcal{N}$ is bounded above by the sum of the positive real parts of the observable eigenvalues. Fix $\varepsilon>0, T>0$ and let $E \subset \bar{K}=\pi(K)$ be a maximal $(T, \varepsilon, \bar{K}, \bar{\Phi})$-separated set with respect to the flow $\bar{\Phi}=e^{\bar{A} \cdot}$ on $\mathbb{R}^{d} / \mathcal{N}$, say $E=\left\{\pi x_{1}, \ldots, \pi x_{n}\right\}$ with $x_{j} \in K$, and let $n:=s(T, \varepsilon, \bar{K}, \bar{\Phi})$. Then $E$ is also $(T, \varepsilon, \bar{K}, \bar{\Phi})$-spanning which means that for all $x \in K$ there is $x_{j}, j \in\{1, \ldots, n\}$, with

$$
\begin{aligned}
\max _{t \in[0, T]} \mathrm{d}\left(e^{\bar{A} t} \pi x, e^{\bar{A} t} \pi x_{j}\right) & =\max _{t \in[0, T]} \operatorname{dist}\left(e^{A t} x-e^{A t} x_{j}, \mathcal{N}\right) \\
& =\max _{t \in[0, T]} \inf _{z \in \mathcal{N}}\left\|e^{A t} x-e^{A t} x_{j}-z\right\|<\varepsilon
\end{aligned}
$$

By assumption, we can assign to each $x_{j} \in K$ a control function $u_{j} \in \mathcal{U}$ such that

$$
\operatorname{dist}\left(\varphi\left(t, x_{j}, u_{j}\right), \mathcal{N}\right)<\varepsilon \text { for all } t \geq T_{0}(\varepsilon) \text {. }
$$

Let $\mathcal{S}:=\left\{u_{1}, \ldots, u_{n}\right\} \subset \mathcal{U}$. Note that $\mathcal{N}+P(Q)=P(Q)$, since for $x_{1} \in \mathcal{N}$ and $x_{2} \in P(Q)$ there is a control $u \in \mathcal{U}$ with

$$
C \varphi\left(t, x_{1}+x_{2}, u\right)=C \varphi\left(t, x_{1}, 0\right)+C \varphi\left(t, x_{2}, u\right)=C \varphi\left(t, x_{2}, u\right) \in P(Q) .
$$

Using $\mathcal{N} \subset P(Q)$ and linearity, we obtain that for all $x \in P(Q)$ there is $j$ such that

$$
\begin{gathered}
\max _{t \in[0, T]} \operatorname{dist}\left(\varphi\left(t, x, u_{j}\right)-\varphi\left(t, x_{j}, u_{j}\right), P(Q)\right) \leq \max _{t \in[0, T]} \operatorname{dist}\left(\varphi\left(t, x, u_{j}\right)\right. \\
\left.-\varphi\left(t, x_{j}, u_{j}\right), \mathcal{N}\right)=\max _{t \in[0, T]} \operatorname{dist}\left(e^{A t} x-e^{A t} x_{j}, \mathcal{N}\right)<\varepsilon
\end{gathered}
$$

Then it follows that for all $t \in\left[T_{0}(\varepsilon), T\right]$

$$
\operatorname{dist}\left(\varphi\left(t, x, u_{j}\right), P(Q)\right)<2 \varepsilon .
$$


In fact, using $\mathcal{N}+P(Q)=P(Q)$, one finds for all $t \in\left[T_{0}(\varepsilon), T\right]$

$$
\begin{aligned}
& \operatorname{dist}\left(\varphi\left(t, x, u_{j}\right), P(Q)\right) \\
& \quad=\inf \left\{\left\|\varphi\left(t, x, u_{j}\right)-\varphi\left(t, x_{j}, u_{j}\right)+\varphi\left(t, x_{j}, u_{j}\right)-p\right\|, \quad p \in P(Q)\right\} \\
& \quad=\inf \left\{\left\|\varphi\left(t, x, u_{j}\right)-\varphi\left(t, x_{j}, u_{j}\right)+\varphi\left(t, x_{j}, u_{j}\right)-p-n\right\|, \quad p \in P(Q) \text { and } n \in \mathcal{N}\right\} \\
& \quad \leq \inf \left\{\left\|\varphi\left(t, x, u_{j}\right)-\varphi\left(t, x_{j}, u_{j}\right)-p\right\|+\left\|\varphi\left(t, x_{j}, u_{j}\right)-n\right\|, \quad p \in P(Q) \text { and } n \in \mathcal{N}\right\} \\
& \quad=\inf \left\{\left\|\varphi\left(t, x, u_{j}\right)-\varphi\left(t, x_{j}, u_{j}\right)-p\right\|, p \in P(Q)\right\}+\inf \left\{\left\|\varphi\left(t, x_{j}, u_{j}\right)-n\right\|, \quad n \in \mathcal{N}\right\} \\
& \quad<\varepsilon+\varepsilon
\end{aligned}
$$

This shows that $\mathcal{S}$ is $\left(T, T_{0}(\varepsilon), 2 \varepsilon, K, Q\right)$-spanning and hence

$$
r_{\mathrm{as}}\left(T, T_{0}, 2 \varepsilon, K, Q\right) \leq s(T, \varepsilon, \pi(K), \bar{\Phi}) \quad \text { for all } T, \varepsilon>0
$$

and consequently

$$
\begin{aligned}
h_{\mathrm{as}}(2 \varepsilon, K, Q) & =\lim _{T_{0} \rightarrow \infty} \limsup _{T \rightarrow \infty} \frac{1}{T} \log r_{\mathrm{as}}\left(T, T_{0}, 2 \varepsilon, K, Q\right) \\
& \leq h_{\mathrm{top}}(\pi(K), \bar{\Phi}) \leq h_{\mathrm{top}}(\bar{\Phi})=\sum_{i: \operatorname{Re} \lambda_{i}>0} \operatorname{Re} \lambda_{i} .
\end{aligned}
$$

In order to combine this result with controllability properties, we show the following lemma which is similar to [6, Theorem 4.1]. (Here, we restrict the analysis to linear control systems, consider asymptotic output invariance entropy instead of invariance entropy, and do not require that $K \subset P$.)

Lemma 5 Consider system (1) and let $K, P \subset \mathbb{R}^{d}$ be nonvoid compact sets with $P$ being controlled invariant. Then, if the Lebesgue measure $\mu(K)$ of $K$ is positive, the following estimate holds:

$$
h_{\mathrm{as}}(K, P) \geq \operatorname{tr} A
$$

Proof Fix $\varepsilon>0$ and $T \geq T_{0}>0$, and let $\mathcal{S}=\left\{u_{1}, \ldots, u_{n}\right\}$ be a minimal $\left(T, T_{0}, \varepsilon, K, P\right)$-spanning set. Define the following sets:

$$
K_{j}:=\left\{x \in K \mid \operatorname{dist}\left(\varphi\left(\left[T_{0}, T\right], x, u_{j}\right), P\right)<\varepsilon\right\}, \quad j=1, \ldots, n .
$$

Then the sets $K_{j}, j=1, \ldots, n$, cover $K$ and, by continuous dependence on initial conditions, the subsets $K_{j}$ are open in $K$. Since $\varphi\left(t, K_{j}, u_{j}\right) \subset \mathbf{N}_{\varepsilon}(P)=\{x \in$ $\left.\mathbb{R}^{d}, \operatorname{dist}(x, P)<\varepsilon\right\}$ for all $t \in\left[T_{0}, T\right]$ and $j=1, \ldots, n$ we obtain, in particular,

$$
\mu\left(\varphi\left(T, K_{j}, u_{j}\right)\right) \leq \mu\left(\mathbf{N}_{\varepsilon}(P)\right) \quad \text { for all } j=1, \ldots, n \text {. }
$$

Moreover, by the transformation theorem and Liouville's formula we get for all $j \in$ $\{1, \ldots, n\}$

$$
\mu\left(\varphi\left(T, K_{j}, u_{j}\right)\right)=\operatorname{det}\left(e^{A T}\right) \cdot \mu\left(K_{j}\right)=\exp (T \cdot \operatorname{tr} A) \cdot \mu\left(K_{j}\right) .
$$


Then it follows that

$$
\mu(K) \leq \sum_{j=1}^{n} \mu\left(K_{j}\right) \leq n \cdot \max _{j=1, \ldots, n} \mu\left(K_{j}\right) \leq n \cdot \frac{\mu\left(\mathbf{N}_{\varepsilon}(P)\right)}{\exp (T \cdot \operatorname{tr} A)}
$$

Consequently, we obtain the estimate

$$
r_{\mathrm{as}}(T, \varepsilon, K, P)=n \geq \frac{\mu(K)}{\mu\left(\mathbf{N}_{\varepsilon}(P)\right)} \exp (T \cdot \operatorname{tr} A)
$$

Taking the logarithm on both sides, dividing by $T$ and letting $T$ tend to infinity yields the inequality

$$
h_{\mathrm{as}}(\varepsilon, K, P) \geq \limsup _{T \rightarrow \infty} \frac{1}{T}\left[\log \mu(K)-\log \mu\left(\mathbf{N}_{\varepsilon}(P)\right)+T \cdot \operatorname{tr} A\right]=\operatorname{tr} A
$$

Letting $\varepsilon$ tend to zero we obtain (16).

The next lemma describes the behavior of the asymptotic output invariance entropy under semiconjugacy. For brevity, we only state and prove the analogue of Theorem 1(i) in the case relevant here.

Lemma 6 Consider for $i=1,2$ two control systems of the form (1),

$$
\dot{x}_{i}=A_{i} x_{i}+B_{i} u(t), y_{i}=C_{i} x_{i}, u \in \mathcal{U},
$$

in $\mathbb{R}^{d_{i}}$ with control range $U \subset \mathbb{R}^{m}$ and outputs $C_{i}: \mathbb{R}^{d_{i}} \rightarrow \mathbb{R}^{k_{i}}$. Let $\pi^{s}: \mathbb{R}^{d_{1}} \rightarrow \mathbb{R}^{d_{2}}$ and $\pi^{\text {out }}: \mathbb{R}^{k_{1}} \rightarrow \mathbb{R}^{k_{2}}$ be linear. Denote the corresponding trajectories by $\varphi_{i}(t, x, u)$ and assume that the following semiconjugacy property holds for all $(t, x, u) \in$ $[0, \infty) \times \mathbb{R}^{d_{1}} \times \mathcal{U}$ :

$$
\pi^{s}\left(\varphi_{1}(t, x, u)\right)=\varphi_{2}\left(t, \pi^{s}(x), u\right) \text { and } \pi^{o u t} \circ C_{1}=C_{2} \circ \pi^{s}
$$

Let $Q \subset \mathbb{R}^{k_{1}}$ be a compact output controlled invariant set for the first system. Then the set $\pi^{\text {out }}(Q) \subset \mathbb{R}^{k_{2}}$ is compact and output controlled invariant. For a compact subset $K \subset \mathbb{R}^{k_{1}}$ the image $\pi^{s}(K) \subset \mathbb{R}^{d_{2}}$ is compact and the asymptotic output invariance entropies for the two systems satisfy

$$
h_{\mathrm{as}}^{1}(K, Q) \geq h_{\mathrm{as}}^{2}\left(\pi^{s}(K), \pi^{\text {out }}(Q)\right) .
$$

Proof The proof of Theorem 1(i) applies literally to show that $\pi^{s}(K)$ is compact and that $\pi^{\text {out }}(Q)$ is compact and output controlled invariant. In the same way, it follows that

$$
\pi^{s}\left(P_{1}(Q)\right) \subset P_{2}\left(\pi^{\text {out }}(Q)\right)
$$


where $P_{1}(Q)$ and $P_{2}\left(\pi^{\text {out }}(Q)\right)$ denote the sets of initial values for the first and the second system leading to outputs in $Q$ and in $\pi^{\text {out }}(Q)$, respectively. Now let $T \geq T_{0}>0$ and $\varepsilon>0$. By linearity, one finds $\delta>0$ such that

$$
\pi^{s}\left(\mathbf{N}_{\delta}\left(P_{1}(Q)\right)\right) \subset \mathbf{N}_{\varepsilon}\left(\pi^{s}\left(P_{1}(Q)\right)\right) \subset \mathbf{N}_{\varepsilon}\left(P_{2}(Q)\right) .
$$

Let $\mathcal{S}_{1} \subset \mathcal{U}$ be a minimal $\left(T, T_{0}, \delta, K, Q\right)$-spanning set for the first system. We claim that it is a $\left(T, T_{0}, \varepsilon, \pi^{s}(K), Q\right)$-spanning set for the second system. In fact, for $x_{2} \in \pi^{s}(K)$ there is $x_{1} \in K$ with $x_{2}=\pi^{s}\left(x_{1}\right)$. Then there is $u \in \mathcal{S}$ with $\varphi_{1}\left(t, x_{1}, u\right) \in \mathbf{N}_{\delta}\left(P_{1}(Q)\right)$ for all $t \in\left[T_{0}, T\right]$. One finds for all $t \in\left[T_{0}, T\right]$

$\varphi_{2}\left(t, x_{2}, u\right)=\varphi_{2}\left(t, \pi^{s}\left(x_{1}\right), u\right)=\pi^{s}\left(\varphi_{1}\left(t, x_{1}, u\right)\right) \in \pi^{s}\left(\mathbf{N}_{\delta}\left(P_{1}(Q)\right)\right) \subset \mathbf{N}_{\varepsilon}\left(P_{2}(Q)\right)$.

This shows that $\mathcal{S}$ is a $\left(T, T_{0}, \varepsilon, \pi^{S}(K), Q\right)$-spanning set. Hence, for every $\delta<\delta(\varepsilon)$, one finds for the minimal cardinalities of spanning sets of the first and the second system that $\left.r_{\mathrm{as}}^{1}\left(T, T_{0}, \delta, K, Q\right) \geq r_{\mathrm{as}}^{2}\left(T, T_{0}, \varepsilon, \pi^{s}(K), Q\right)\right)$, and inequality (17) follows.

Combining Theorem 4 with controllability properties, we obtain the following characterization of the asymptotic invariance entropy.

Theorem 5 Suppose, in addition to the assumptions of Theorem 4, that $0 \in \operatorname{int} U$ and that $K$ is a compact subset of the reachability subspace $\mathcal{R}$ which contains the origin in its interior. Assume, furthermore, that there are no reachable and observable eigenvalues on the imaginary axis. Then the asymptotic output invariance entropy satisfies

$$
h_{\mathrm{as}}(K, Q)=\sum_{\lambda} \operatorname{Re} \lambda
$$

where the sum on the right-hand side is taken over all observable and reachable eigenvalues $\lambda$ with positive real parts.

Proof (i) For $x \in \mathcal{R}$ and $u \in \mathcal{U}$ one has $\varphi(t, x, u) \in \mathcal{R}$ for all $t \geq 0$. This shows that $P(Q) \cap \mathcal{R}=P_{\mathcal{R}}(Q):=\{x \in \mathcal{R}$, there is $u \in \mathcal{U}$ with $C \varphi(t, x, u) \in Q$ for all $t \geq 0\}$.

Since $K \subset \mathcal{R}$ it follows for $t>0, x \in K$, and $u \in \mathcal{U}$ that

$$
\varphi(t, x, u) \in \mathbf{N}_{\varepsilon}(P(Q)) \text { implies } \varphi(t, x, u) \in \mathbf{N}_{\varepsilon}(P(Q)) \cap \mathcal{R}=\mathbf{N}_{\varepsilon}\left(P_{\mathcal{R}}(Q)\right) .
$$

It follows that a $(T, \varepsilon, K, Q)$-spanning set of the system with state space restricted to $\mathcal{R}$ is also $(T, \varepsilon, K, Q)$-spanning for the original system. Thus $h_{\text {as }}(K, Q)$ is less than or equal to the corresponding invariance entropy for the system restricted to $\mathcal{R}$. Then Theorem 4 implies that $h_{\text {as }}(K, Q)$ is bounded above by the sum in (18).

(ii) For the other inequality, consider the projection $\pi^{s}$ to the induced observable system and take for $\pi^{\text {out }}$ the identity. By Lemma 6

$$
h_{\mathrm{as}}^{1}(K, Q) \geq h_{\mathrm{as}}^{2}\left(\pi^{s}(K), Q\right) .
$$


Next restrict the observable system to its reachability subspace $\overline{\mathcal{R}}$. Since $0 \in \operatorname{int} K$, it follows that $0 \in \operatorname{int} \pi^{s}(K)$ and hence 0 is also in the interior of $\pi^{s}(K) \cap \overline{\mathcal{R}}$; in particular, this set has nonvoid interior. Clearly, the invariance entropies satisfy

$$
h_{\mathrm{as}}^{2}\left(\pi^{s}(K), Q\right) \geq h_{\mathrm{as}}^{3}\left(\pi^{s}(K) \cap \overline{\mathcal{R}}, Q\right),
$$

where the right-hand side denotes the asymptotic output invariance entropy for the system restricted to $\overline{\mathcal{R}}$. It is immediate from the definitions that the asymptotic output invariance entropy satisfies

$$
h_{\text {as }}^{3}\left(\pi^{s}(K) \cap \overline{\mathcal{R}}, Q\right)=h_{\text {as }}^{4}\left(\pi^{s}(K) \cap \overline{\mathcal{R}}, P(Q)\right),
$$

where on the right-hand side we consider the asymptotic invariance entropy in the state space. Since this is an observable system, the corresponding set $P(Q)$ is compact by Lemma 3(ii). Now we can apply Lemma 5 to estimate $h_{\text {as }}^{4}\left(\pi^{s}(K) \cap \overline{\mathcal{R}}, P(Q)\right)$ from below: If the linear map $\left.\bar{A}\right|_{\overline{\mathcal{R}}}$ induced by $A$ for the observable system restricted to $\overline{\mathcal{R}}$ is totally unstable (i.e., all eigenvalues have positive real parts), the assertion immediately follows. Otherwise, one has to project this system to its unstable part along the center-stable subspace. Since, again, the asymptotic invariance entropy does not increase, the assertion also follows in the general case (see Step 3 in the proof of [6, Theorem 5.1] for details of these arguments).

\section{References}

1. Allgöwer F, Zheng A (eds) (2000) Nonlinear model predictive control. In: Progress in systems and control theory, vol 26. Birkhäuser Verlag, Basel

2. Antsaklis P, Michel A (2006) Linear systems. Birkhäuser, Boston

3. Bowen R (1971) Entropy for group endomorphisms and homogeneous spaces. Tran Am Math Soc 153:401-414

4. Carli R, Bullo F (2009) Quantized coordination algorithms for rendezvous and deployment. SIAM J Control Optim 48:1251-1274

5. Colonius F (2010) Minimal data rates and invariance entropy. In: Electronic proceedings of the conference on mathematical theory of networks and systems (MTNS), Budapest July 5-9

6. Colonius F, Kawan C (2009) Invariance entropy for control systems. SIAM J Control Optim 48:17011721

7. Colonius F, Kliemann W (2000) The dynamics of control. Birkhäuser, Boston

8. Colonius F, Spadini M (2003) Uniqueness of local control sets. J Dyn Control Syst 9:513-530

9. Grüne L (2009) Analysis and design of unconstrained nonlinear MPC schemes for finite and infinite dimensional systems. SIAM J Control Optim 48:1206-1228

10. Gupta V, Dana A, Hespanha J, Murray R, Hassibi B (2009) Data transmission over networks for estimation and control. IEEE Trans Autom Control 54:1807-1819

11. Katok A, Hasselblatt B (1995) Introduction to the modern theory of dynamical systems. Cambridge University Press, Cambridge

12. Kawan C (2009) Invariance entropy for control systems. Doctoral thesis, Institut für Mathematik, Universität Augsburg, 2009. http://opus.bibliothek.uni-augsburg.de/volltexte/2010/1506/

13. Lee EB, Markus L (1967) Foundations of optimal control theory. Wiley, New York

14. Macki J, Strauss A (1982) Introduction to optimal control theory. Springer, Berlin

15. Nair G, Evans RJ, Mareels I, Moran W (2004) Topological feedback entropy and nonlinear stabilization. IEEE Trans Autom Control 49:1585-1597 
16. Robinson C (1999) Dynamical systems. stability, symbolic dynamics, and chaos, 2nd edn. CRC Press, West Palm Beach

17. Wong W, Brockett R (1997) Systems with finite communication bandwidth constraints.I. State estimation problems. IEEE Trans Autom Control 42:1294-1299

18. Wong W, Brockett R (1999) Systems with finite communication bandwidth constraints II. Stabilization with limited information feedback. IEEE Trans Autom Control 44:1049-1053 